\title{
Development of ferroelectric domains and topological defects in vacancy doped ceramics of $\mathrm{h}-\mathrm{LuMnO}_{3}$
}

\author{
A. Baghizadeh, ${ }^{1)}$ J. M. Vieira, ${ }^{1)}$ P. Mirzadeh Vaghefi, ${ }^{2)} \mathrm{M}-\mathrm{G}$. Willinger, ${ }^{3)} \mathrm{V} . \mathrm{S}$. \\ Amaral ${ }^{2)}$ \\ ${ }^{1}$ Department of Materials \& Ceramic Engineering \& CICECO, Aveiro University, 3810-193 Aveiro, Portugal \\ ${ }^{2}$ Physics Department \& CICECO, Aveiro University, Aveiro, Portugal \\ ${ }^{3}$ Department of Inorganic Chemistry, Fritz Haber Institute of the Max Planck Society, Faradayweg 4-6, Berlin, \\ Germany
}

Self-doping of $h-\operatorname{LuMn}_{\mathrm{x}} \mathrm{O}_{3 \pm \delta}(0.92 \leq \mathrm{x} \leq 1.12)$ phase and changes of sintering time are applied to investigate the formation and annihilation of antiphase ferroelectric (FE) domains in bulk ceramics. The increasing of annealing time in sintering results in growth of FE domains which depends on type of vacancy, 6-fold vortices with dimensions of the order of 20 microns being observed. Interference of planar defects of the lattice with the growth of topological defects shows breaking of 6-fold symmetry in the self-doped ceramics. The role of grain boundaries on the development of topological defects has studied. Dominance of the atypical FE domain network in very defective h- $\mathrm{LuMn}_{\mathrm{x}} \mathrm{O}_{3 \pm \delta}$ lattices saturated with Mn vacancies $(x<1)$ was also identified in the current study. After long annealing time scattered closed-loops of nanodimensions are often observed isolated inside large FE domains with opposite polarization. Restoring of the polarization after alternative poling with opposite electrical fields is observed in FE domains. Stress/strain in the lattice driven either by planar defects or chemical inhomogeneity results in FE polarization switching in nano scale and further formation of nano-vortices, detailed investigation having been carried out by electron microscopy. Pinning of FE domains to planar defects is explored in the present microscopy analysis and nano-scale observation of lattices is used to explain features of the ferroelectricity revealed in PFM images of the ceramics.

\section{Introduction}

Ferroelectricity of hexagonal h- $\mathrm{RMnO}_{3}$ oxides presents strikingly new features of most relevance for this class of multiferroics as they show intrinsic interlocking of topological defects the anti-phase boundaries (APB), with ferroelectric (FE) domain walls. The underlying physics of interlocking of FE domains with topological defects in the lattices of single crystals of h- $\mathrm{RMnO}_{3}$ oxides is intensively investigated ${ }^{1-6}$. Attention recently paid to patterns of APBFE domain walls interacting with oxygen vacancies or point defects in the crystalline lattices of h- $\mathrm{RMnO}_{3}$ oxides different from 6-fold vortex networks of stoichiometric composition created better understanding of mutual interaction of lattice defects and FE domains in these oxides. Still, studies on the role of vacancies with effective electric charge, planar 
defects and dislocations on the FE properties and formation of vortex/antivortex domain patterns in these oxides are rare 7,8 .

Evidence of the effects of Lu vacancies, or Mn vacancies and extended defects of the crystalline lattice on FE domains of as sintered $\mathrm{h}-\mathrm{LuMn}_{\mathrm{x}} \mathrm{O}_{3 \pm \delta}$ ceramics will be presented in this paper. Cation vacancies of $\mathrm{Mn}(\mathrm{x}<1)$ or $\mathrm{Lu}(\mathrm{x}>1)$ are introduced by self-doping the h- $\mathrm{RMn}_{\mathrm{x}} \mathrm{O}_{3}$ oxide as a way to alter the unit cell dimensions and perturb ion-to-ion interactions with shifts away from stoichiometric composition. The evolution of FE domains and 6-fold vortices and the causes of topology breaking in self-doped ceramics were investigated by means of PFM and TEM/STEM analysis. The effects of annealing time as a parameter of the sintering process on the FE domain growth and rendering of 6-fold vortices were also studied. TEM analysis is used to reveal interactions of planar defects at the nano-scale or shifts of elemental composition within FE domains. Dark-field TEM with lattice images taken around defective regions in the correct crystal orientation to project the displacement of $\mathrm{Lu}$ ions is employed to explain interactions of planar defects with shifting of polarization switching/phase on domain walls.

\section{Topological networks}

The essential feature of topological FE domains in polar h- $\mathrm{RMnO}_{3}$ derives from their distribution around 6-fold vortices in space. As seen at room temperature, the core of each vortex in complete 6-fold domain topology is the intersection of volumes of three anti-phase domains with $2 \pi / 3$ phase shifts, the direction of FE polarization being reversed when the vortex-core line is crossed inside the same anti-phase domain with equal trimerization angle ${ }^{2,3,9,10}$. Vortex-core lines of complete 6-fold vortex networks in h- $\mathrm{RMnO}_{3}$ perfect crystals are free to bend in single or multiple U-turns; they may even form closed-loops but never intersect together inside the crystal, their free ends being found on crystal surfaces. On the same principle nervures of several vortex-core lines of the set of APB-FE domain walls delimiting the volume of one given FE domain in no way intersect together ${ }^{2,11}$. Each vortex-core line links one vortex with clockwise rotation of $\alpha, \beta$ and $\gamma$ antiphase domains to the corresponding antivortex with anticlockwise rotation of the same antiphase domains. Closed-loop vortices of 6-fold topology hold the edges of the inmost lenticular FE domain volume which has same trimerization angle but the displacement of $\mathrm{R}_{2}$ ions (with $4 \mathrm{~b}$ Wyckoff position) and hence FE polarization symmetrical to those of the outer antiphase FE domain that contains the loop. The edges of the four caped volumes of the remaining FE domains with antiphase and polarization directions given by the rules of 6-fold vortices also meet on the closed-loop vortex-core line ${ }^{7,10,12,13}$. The inversion of $\mathrm{R}_{2}$ ions displacement and hence of electric polarization at APB-FE domain walls in h- $\mathrm{RMnO}_{3}$ is abrupt and occurs within a single (010)a (Type-I APB-FE wall) or double (020)a (Type-II) plane spacing taken in the original $\mathrm{P}_{3} / \mathrm{mmc}$ lattice of the hexagonal apolar h- $\mathrm{RMnO}_{3}$ phase. The specific surface energy of the 
APB-FE domain walls is very low and has lowest estimated values of $10.4 \mathrm{mJm}^{-2}$ and $11.5 \mathrm{mJm}^{-2}$ when they coincide with the $\{210\}$ and $\{120\}$ planes of the $\mathrm{P}_{3} \mathrm{~cm}$ h- $\mathrm{RMnO}_{3}$ lattice respectively ${ }^{3,12}$. The dimension of vortex-core diameter has not been established on first principles. But, outputs of Monte Carlo simulations, atomic models for local distortion of ion positions where the six APB-FE boundaries in cover-leaf configuration meet together and direct observation of vortex cores by HAADF in $\mathrm{C}_{\mathrm{s}}$-corrected STEM microscopy represent the region of intersection of APB-FE interfaces also sharp. It may range from one to two $\mathrm{P}_{3} \mathrm{~cm}$ unit cell sizes, or up to five unit cell dimensions if one accounts for the relaxation in the region around vortex-core central lines within the limit of detection of displacements of the $\mathrm{R}^{3+}$ ions at the frontier of present state-of-art electron microscopy. This is also the range of closest distances two APB-FE walls can approach together pressed by the poling of applied electrical fields or by self-poling from gradients of electrochemical potential created at the surfaces of the h- $\mathrm{RMnO}_{3}$ materials. Such narrow spacing of FE walls and diameters of vortexcores remain below the best lateral resolution obtained in piezoresponse force microscopy in ambient air $6,9,14-18$.

In the tension field side of the edge dislocation with Burgers vector $\mathbf{b}=[100]_{\mathrm{a}}$ and $\{001\}_{\mathrm{a}}$ slip planes taken in the apolar phase the slip strain is distributed by elastic deformation in a distance of approximately ten (020)a plane spaces, the slip vector being equivalent to $2 \pi / 3$ phase shift in trimerization displacement ${ }^{7,12,19}$. The stress field around the edge dislocation is asymmetrical. At the transition temperature $\mathrm{T}_{\mathrm{s}}$ when the polar phase of h- $\mathrm{RMnO}_{3}$ phase forms by trimerization of the apolar phase the free energy for vortex creation close to the edge dislocation is locally modified and single dislocation truncated-vortices appeared with just 4-state topology locked to the edge dislocations. If two identical edge dislocations with Burgers vector $\mathbf{b}=[100]_{\mathrm{a}}$ in neighbor $\{001\}_{\mathrm{a}}$ slip planes become associated within a narrow distance comparable to the range of vortex-core diameters the total Burgers vector given by the accumulated slip strain becomes $2 \mathbf{b}=[200]$ and a double-dislocation truncated-vortices with 2 -state topology and $4 \pi / 3$ trimerization displacement is generated locked to the dislocation pair ${ }^{7,17,20}$. Dislocations and vortex-core lines can both bend inside crystals. Locking vortex-cores to the dislocations of Burgers vector $\mathbf{b}=[100]_{\mathrm{a}}$ does not have to be lost if the dislocations become mixed or screw dislocations at places where dislocation lines change their orientation. Contrary to 6-fold vortexcore lines dislocations can react forming nodes of three dislocations composing poorly organized networks, the Frank net. Rows of dislocations form tilt boundaries and low-angle grain boundaries in bulk materials as well as in flawed single crystals. Fine precipitates pin dislocations and hinder dislocation glide. Post-recoalescence temperature $T_{p}$ of rapidly solidified $\mathrm{RMnO}_{3}$ compounds gives approximately values of their melting points. Values of $\mathrm{T}_{\mathrm{p}}$ have a moderate increase with Shannon $\mathrm{R}^{3+}$ ionic radius from $\mathrm{T}_{\mathrm{p}}=1910 \mathrm{~K}$ in $\mathrm{HoMnO}_{3}$ to $\mathrm{T}_{\mathrm{p}}=1960 \mathrm{~K}$ in $\mathrm{LuMnO}_{3}{ }^{21}$. Ductile deformation by creep and activation of primary slip systems of the $\mathrm{h}-\mathrm{RMnO}_{3}$ lattice under applied load was observed in the range of homologous temperature of $0.72-0.75$ of the melting point overlapping with transition temperature, $\mathrm{T}_{\mathrm{s}}$. Interaction of created vortices with shear strain of the lattice undergoing plastic deformation under load pulls vortices and antivortices 
in opposite directions, moves them in parallel with travelling dislocations gliding in primary slip planes resulting in long topological stripes closely aligned to slip bands. Interaction of vortex-cores and dislocations represents a complex way of mechanical control of topological defects of the h- $\mathrm{RMnO}_{3}$ multiferroics. Vortex-cores that otherwise remain immobile for temperatures significantly below $\mathrm{T}_{\mathrm{s}}$ can react to poling with electric fields one order of magnitude above the coercive field or to local heating from lightning discharges at the crystal edges and move towards the edges of the crystal leaving stripe domain patterns behind ${ }^{18,22-24}$.

The temperature of apolar-to-polar phase transition $\mathrm{T}_{\mathrm{s}}$ of $\mathrm{h}-\mathrm{RMnO}_{3}$ is directly proportional to Shannon $\mathrm{R}^{3+}$ ionic radius and increases from $\mathrm{T}_{\mathrm{s}}=1373 \mathrm{~K}$ in h-HoMnO to $\mathrm{T}_{\mathrm{s}}=1672-1708 \mathrm{~K}$ in h-LuMnO , the value of $\mathrm{T}_{\mathrm{s}}$ for $\mathrm{h}-\mathrm{YMnO}_{3} \mathrm{~T}_{\mathrm{s}}=$ $1220-1260 \mathrm{~K}$ being roughly $70 \mathrm{~K}$ below the corresponding trend value determined from the dependence of $\mathrm{T}_{\mathrm{s}}$ on ionic radius of the other $\mathrm{h}-\mathrm{RMnO}_{3}(\mathrm{R}=\mathrm{Ho}-\mathrm{Lu})$ oxides ${ }^{5,11,17,25,26}$. Up to a threshold value of cooling rate h- $\mathrm{RMnO}_{3}$ samples annealed above $\mathrm{T}_{\mathrm{s}}$ and cooled in a controlled way present a density of vortex-core lines (length/volume or intersection points/area) and hence the specific surface area of APB-FE walls and properties their dependent which increases in proportion to a non-integer power of cooling rate. This threshold of cooling rate that separates the Kibble-Zurek mechanism (low cooling rates) from the anti- Kibble-Zurek regime (at high cooling rates) with the opposite trend moves to higher values with the decreasing of $\mathrm{R}^{3+}$ ionic radius from the $\mathrm{Y}^{3+}$ to $\mathrm{Tm}^{3+}$ implying a large decreasing of vortex-core densities for the smaller ionic radius at cooling rates in the range of $1 \mathrm{Kmin}^{-1}$. The highest values of vortex-core density of so-called Kosterlitz - Thouless (KT) phase, or Higgs phase, in 6-fold topological networks reported for h- $\mathrm{RMnO}_{3}$ single crystals at the limits of Kibble-Zurek regime range to 0.6 vortex. $\mu \mathrm{m}^{-2}$ corresponding to average vortex-core spacing of $1.35 \mu \mathrm{m}^{5,11,17}$. Samples processed or annealed in the narrow temperature range below $\mathrm{T}_{\mathrm{s}}$ develop as equilibrium phase curved loop domains, or Coulomb phase, the annular domain patterns being made of layers of antiphase domains separated by topological APB-FE walls wrapped around the central toroidal volume without a vortexcore. For processing temperatures below 0.95 of $\mathrm{T}_{\mathrm{s}}$ the Ising-type stripe domain patterns with straight APB-FE walls, that may become curved near the limit of stability of this mechanism, are expected to form as the stable configuration of the APB-FE domains of $\mathrm{h}-\mathrm{RMnO}_{3}$ phases ${ }^{11,17}$. Most of bulk h- $\mathrm{RMnO}_{3}$ materials processed by sol-gel methods or by solidstate synthesis from mixture of oxides, thin films and even thin plate-like single crystals grown using flux methods were processed at temperatures below the corresponding $\mathrm{T}_{\mathrm{s}}$ temperatures and with cooling rates which are rarely controlled or reported. Adding to the multiplicity of topology patterns of APB-FE domain networks potentiated in the $\mathrm{h}-\mathrm{RMnO}_{3}$ lattices, the fine grain sizes of polycrystalline materials may easy fall below the scale of core-vortex average distance in equilibrium. Due to anisotropy of APB-FE domain wall specific surface energy of the FE domain walls of $\{210\}$ and $\{120\}$ prismatic planes as well as of the $\{001\}$ basal planes are the most probable and offer privileged orientations for their observation because they are perpendicular to the image plane when seen in [100] and [110] zone axis of TEM and 
STEM microscopy, and the formers are also normal to the basal $\{001\}$ planes, the most extensive face of flux-grown single crystals and become exposed by selective chemical etching ${ }^{7,13,17-19,23,27}$. Besides extrinsic effects created on FE domain networks by dislocations, defect clusters and grain boundaries the random orientation of grains in relation to the surface poses additional complexities in the observation of APB-FE domains of polycrystalline samples. Cut at random the crystalline grains in polished cross-sections of bulk h- $\mathrm{RMnO}_{3}$ prepared for PFM examination display inclined domain walls in larger proportions than in preferable orientations for their observation with domain walls perpendicular to image planes. Overlapping of FE domains in thin slabs of FE domains of up to $2 \mu \mathrm{m}$ thickness softens PFM contrast and blurs the traces of sloped domain walls, the fuzziness being more confusing if occurring near vortex-cores. If such constraint may easy be lifted in TEM and HAADF/STEM studies of $\mathrm{h}-\mathrm{RMnO}_{3}$ bulk samples by using electron diffraction to find thin sections of grains with correct orientation, in polished cross-sections of polycrystals for PFM examination grain orientation can only be determined ex-situ by using EBSD in SEM, the spatial resolution of the EBSD comparing with resolution limits of PFM ${ }^{6,14,28,29}$.

\section{Experiment}

$\mathrm{LuMn}_{\mathrm{x}} \mathrm{O}_{3 \pm \delta}$ ceramics $(0.92 \leq \mathrm{x} \leq 1.12)$ were prepared by conventional solid state sintering of properly mixed $\mathrm{Lu}_{2} \mathrm{O}_{3}$ and $\mathrm{MnO}_{2}$ oxide powders with $99.99 \%$ purity. To increase homogeneity of composition high temperature annealing in air was performed in three steps, one day firing at each of the isothermal holds $850{ }^{\circ} \mathrm{C}, 1000{ }^{\circ} \mathrm{C}$ and $1300{ }^{\circ} \mathrm{C}$ with intermediary grinding and pressing of samples into pellets, the time of the last firing step at $1300^{\circ} \mathrm{C}$ having been extended to 5 days or 10 days to determine the effect of annealing time ${ }^{30}$. The grain size and composition change were both checked in SEM/EDS. SEM images have shown that by increasing $x$ value, there is an increase in grain size from 1.78 microns $(\mathrm{x}=0.92)$ to 3.23 microns $(\mathrm{x}=1.08)$ for fixed time of one day annealing. Increase in grain size is also observed while the annealing time increases from one day to 10 days, for example for sample $\mathrm{x}=1.08$, after 10 days annealing, grain size reaches to average of 8.1 microns. Change in the volume of the unit cell versus $\mathrm{x}$ value shows sensitivity of the unit cell to the off-stoichiometric in our study (Supplementary Materials, Figure A1). NT-MDT NTEGRA PRIMA modular sensor force microscope was used for AFM/PFM measurements at room temperature in contact mode (10 V AC voltage and $50 \mathrm{kHz}$ frequency on the tip). Cantilevers from NanoSensor Co., Pointprobe-Plus Silicon-SPM-Sensor made of $\mathrm{n}^{+}$-doped silicon coated by Al on the detector side with tip height of 10-15 microns and 10 $\mathrm{nm}$ tip radius were used for all measurements. Flat surfaces of the ceramics were prepared by mechanical polishing down to 0.1 micron lapping paper. Polished samples were heated at $130^{\circ} \mathrm{C}$ to eliminate surface humidity before PFM experiments. Thin sections for TEM study were prepared by conventional mechanical polishing and precision ion milling 
down to electron transparency. JEOL JEM2200FS FEG $200 \mathrm{kV}$ TEM/STEM equipped with the Omega filter and EDSINCA $\mathrm{x}$-sight detector was used for TEM. HRTEM images were energy filtered using $10 \mathrm{eV}$ energy slit. HAADF-STEM images were taken in the dedicated STEM Hitachi HD2700 equipped with EDS Bruker and nano-diffraction, operated at $200 \mathrm{kV}$.

\section{Results and Discussion}

Fig.1.a shows the effect of annealing time at $1300^{\circ} \mathrm{C}$ on evolution of vortices in vacancy-doped samples and in the one of stoichiometric composition. The piezoresponse of the two compositions of $x=1.08$ (one day annealing, not shown here) and $x=1.04$ (10 days annealing, Fig.1.a) reveals change in size of 6-fold vortices. Fig.1.b shows a 6-fold vortex observed in the sample of stoichiometric composition $(x=1.00)$ annealed for 5 days. In the case of short annealing time the ferroelectric domain pattern exhibits a type-II vortex networking with preferred polarization direction downwards ${ }^{2,31}$. Type-II like vortex patterns are not unique in samples of $x>1$, short annealed, also type-I vortex patterns without dominant polarization direction were observed in same samples. Vortex pattern dimensions in sample of $x=1.08$, short time annealing are roughly below 8 microns. Observation of wider 6-fold vortex of type-I in Fig.1.a with size close to 20 micron illustrates the effect of annealing time on scale of 6 -fold vortices. Grain sizes of current ceramics increased with annealing time and samples of heat treated for longer time sustain larger FE domains. However, the size of vortex/antivortex in Fig.1.a goes beyond the estimated grain size in the range of 8 microns for this sample. Within the resolution limit of PFM apparatus, vortex core fragmentation of 6-fold vortices is found which had been shown to be a feature of vortex patterns in either a-c or b-c planes of the hexagonal lattice ${ }^{1,13}$. Core fragmentation in $\mathrm{h}-\mathrm{YbMnO}_{3}$ single crystals was discussed because the presence of same trimerization antiphase resulted in existence of charged head-to-head domain walls of high energy while in present case the phase map in the area of the bulk material analyzed by PFM is incomplete and insufficient to indicate head-to-head domain walls in Fig.1.b ${ }^{31}$.

Fig.1.c displays FE domains of sample $\mathrm{x}=0.92$ after long annealing time (10 days). Left part of Fig.1.c shows 6 -fold vortices where upward polarization (bright region) occupies lesser area than regions of downward polarization. Nonuniform distributions of upwards and downwards polarized FE domains give rise to type-II vortex pattern ${ }^{31}$. Present investigation of samples with either Mn vacancies, or Lu vacancies and of stoichiometric composition annealed for different time could only find 6-fold vortices of type-I in samples with $x \geq 1$. Introducing Mn vacancies $(x<1)$ likely changed the type of FE domain network to the type-II pattern regardless of how long annealing time takes. Three stripelike FE domains with alternation of polarization and phase are observed in right part of Fig.1.c and d while their 
propagation cannot be continued further. The topography map of the same region revealed that spread of topological defects is interrupted at the grain boundaries. Elongation of the three FE domains observed in this figure does not replicate the geometry of 6-fold symmetry of FE domains, showing type II FE walls. Breaking of 6-fold symmetry driven by chemical composition of the gaseous atmosphere was reported in Ar-grown (oxygen deficient) single crystal $\mathrm{YMnO}_{3-\delta}$, whereas the air-grown single crystal retains the regular topology of the FE domains ${ }^{32,33}$. Type-II domain walls were found near the free surface of $\mathrm{h}-\mathrm{RMnO}_{3}$ single crystals after electrical poling with applied electrical fields or due to self-poling by the electrical field component of total electrochemical potential that drives interdiffusion of ionic species at high temperature resulting from shifts of oxygen activity in the atmosphere ${ }^{2,31}$. In samples with nominal concentration of $0.08 \% \mathrm{Mn}$ vacancies the shift in chemical composition can be considered as most probable reason of promoting appearance of stripe-like FE domains.

Grain boundaries and pores shall affect the mechanisms of FE domain growth in bulk ceramic materials. Their effects had not been comprehensively studied in h- $\mathrm{RMnO}_{3}$ oxides, yet. The white circle in the right of the Fig.2.a indicates the position of the triple line where grain boundaries met (topography map of the same region is provided in Fig.2.c). Local curvature of the grain boundaries is determined by equilibrium of surface tension forces. Fig.2.a shows a second example of FE domains in broken 6-fold symmetry, where three of the FE domains with opposite polarization directions and different phases emerge from the clamping point on the wall of a large FE domain in the neighbor crystalline grain (the black, dashed line). The three FE domains creating the truncated vortex in Fig.2.a belong to the same crystalline grain of the bulk ceramics (more figures of the same region in Supplementary Materials, Figure A3). The formation of a complete 6-fold vortex was hampered upon meeting the grain boundary (indicated by dashed black line). Missing 6-fold symmetry of the vortex is also observed in the stoichiometric sample ( $\mathrm{x}=1.00,5$ days annealing) in Fig.2.b inside a large grain where topology map assures absence of grain boundaries. Vanishing of one FE domain in neighboring FE domains of opposite polarization is seen on the left, down part of Fig.2.b where a 6-fold vortex would be expected. Inset of Fig.2.b gives the smoothed profile intensity of voltage taken across the marked line in same figure. The topography map of the same region is shown in Fig.2. d. The intensity profile in the phase map and in amplitude map (not shown) reveals the trace of the fading FE domain with downward polarization limited by faint walls in a narrow width of about $300 \mathrm{~nm}$. Compared to oxygen deficient crystals, Fig.2.b does not represent type-II FE domain or atypical domain patterns of vacancy doped $\mathrm{h}-\mathrm{RMnO}_{3}$ single crystals. Instead, weakening of the downward $\mathrm{FE}$ domain can be assigned to canting of vortex core with inclined domain walls or to dislocations in the lattice to be discussed later.

Fig.2.e and d reveals dispersion of nano-sized FE domains inside wider domains with opposite polarization. This scenario of dispersive nano-regions possessing different phase shift or polarization direction than the parent FE domain surrounding them was also observed in samples of other compositions in this study. Upward polarization (bright regions) 
is dominant in a wide extension displayed in Fig.2.e, whereas regions of downward polarization form discrete patches. From the region of downward polarization in the inset of Fig.2.e the image of higher magnification in Fig.2.f clearly confirms the presence of nano-FE domains inside larger FE domains with opposite polarization.

To shed light on interaction of FE domains of self-doped ceramics and applied electric field, PFM images were taken before and after electrical poling of the region shown in Fig. 3.a of sample $\mathrm{x}=1.04,10$ days annealing. Fig. 3.a presents a set of narrow FE domains of negative (darker contrast) and positive (brighter contrast) polarization. Polling was done by applying $+30 \mathrm{~V}$ external potential on the region of Fig. 3.b where a narrow negative domain is surrounded by two domains of positive polarization. As clearly seen in Fig. 3.c of the poled area, applied field increased the width of the negative domain and made diffuse the domain walls. Reversibility of the FE domain was tested by applying a negative voltage $(-30 \mathrm{~V})$ on this same region. The original FE configuration of the Fig. 3.b before poling experiment is reproduced back in Fig. 3.d after reverse poling, although contrast is slightly changed. Profiles of PFM response intensity along the arrow lines before poling and after successive poling experiments are compared in the inset of Fig. 3.d. Width broadening of the negative domain from the profile after $+30 \mathrm{~V}$ poling and almost complete recovery of the negative domain after $-30 \mathrm{~V}$ poling are visible. Positive poling reduces the intensity of positive domains, whereas the intensity of the negative domain under positive or negative poling remains the same as before poling. This experiment and similar experiments on other samples show pinning of negative domains upon applying a positive field which is restored by changing the polarization of the electrical field. Restoring of the original polarization of the FE domains after poling shown in Fig.3 indicates that memory effect of $\mathrm{FE}$ domains exists in self-doped $\mathrm{h}-\mathrm{RMnO}_{3}$ ceramics.

Electron microscopy analysis of defective regions was performed to characterize the interactions of defects and ferroelectricity in the lattice of $\mathrm{h}-\mathrm{RMnO}_{3}$ ceramics. The high magnification DF-TEM image in Fig.4.a shows the complete 6-fold vortex pattern of size in the range of $150 \mathrm{~nm}$ with indication of the phase shift and polarization of the FE domains found in sample $\mathrm{x}=1.04$ on the edge of the particle in the inset of Fig.4.b. Fig.4.b is the HAADF-STEM image of this particle in the right side of the vortex. Displacement of the Lu ions as two up one down corresponds to upward polarization of the FE domain. The nano-vortex is located at the end of a planar defect, the defect being extended to the inside of the particle. The planar defect splits in two branches near the edge of the particle at a distance roughly $200 \mathrm{~nm}$ away from vortex core. The DF-TEM image of the planar defect in the inset of Fig.4.b was taken by selecting the 004 diffraction spot with the particle aligned to $<110>$ zone axis. EDS analysis did not reveal any change of composition on scanning domain walls between FE domains.

The STEM image of two planar defects converging to the point on the edge of a second particle of same sample is shown in Fig.5.a. In inset of Fig.5.a the EDS analysis across one of the planar defect, marked with the arrow, shows deviation of 
$\mathrm{x}=\mathrm{Mn} / \mathrm{Lu}$ ratio from the $\mathrm{x}=1.08$ average composition on crossing the defective region. To exclude the contribution of copper from sample holder in the EDS analysis the $\mathrm{Mn} / \mathrm{Lu}$ ratio was calculated from the intensities of $\mathrm{K}_{\alpha 1} \mathrm{Mn}$ line and $\mathrm{L}_{\alpha 1}$ of Lu line. The HRTEM image of the lattice around the planar defect with the particle aligned to the $<110>$ zone axis in Fig.5.b exhibits changes in the structure of the lattice revealing presence of one anti-phase boundary marked by two yellow lines. One cannot determine polarization switching across the planar defect. Interference of extended defects of the crystal with topological defect can affect appearance of 6-fold vortices, giving rise to vortices with even sub-six topological orders ${ }^{7,8}$.

Switching of FE polarization near an extended defect is seen in the TEM image along [110] zone axis of sample $\mathrm{x}=1.00$, ( 5 days annealing) in Fig.5.c where contrast and profiles of intensity are used to pin point the planes of the APB-FE domain walls with switching of phase of Lu ion displacement. The same criteria were used to determine the phase shift of the two neighboring FE domains ${ }^{2,7}$. Inset of Fig.5.c displays the low magnification TEM image of the defective region and lattice around it. Lines 1 and 2 in Fig.5.c show Lu rows under the influence of the defect. Intensity profiles were determined along these lines, plots in Fig.5.d, with contrast heights indicating the position and displacements of Lu ions. The sharp switching of polarization pitch in the intensity profiles gives the position and type of the APB-FE walls. Both APB-FE walls crossed by lines 1 and 2 respectively are of type-II with one apolar ion plane separating antiphase FE domains of opposite polarity. The appearance of the APB-FE domain walls seems to emerge from the strain field created in the lattice by the defect. The strain/stress effect on topological defects in single crystals of h-ErMnO ${ }_{3}$ was recently investigated ${ }^{7,8,23}$. Shear strain of applied loads on the h- $\mathrm{RMnO}_{3}$ crystals at high temperature force movement of vortices along the planes of maximum shear strain rate with anti-vortices moving in opposite direction to the corresponding vortices. By the effect of coordinated movements the FE domains are stretched to topological stripes with FE domain walls preferentially aligned along the primary slip planes, the $\{110\}$ planes of the hexagonal lattice ${ }^{23}$. Lattice may show changes of FE wall type on the transition region from the 6-fold vortices to stretched-stripe FE domains ${ }^{23}$. The interaction of topological defects with extended defects namely the partial edge dislocations in $\mathrm{Y}_{0.67} \mathrm{Lu}_{0.33} \mathrm{MnO}_{3}{ }^{7}$ or $\mathrm{YMnO}_{3}{ }^{8}$ single crystals generated unconventional features of FE vortices namely low even order vortices: 4-fold vortex or single dislocation truncated vortex and 2-fold vortex, or a double dislocation truncated vortex. As shown in Fig.5.c nano-regions of opposite polarization and different phase are formed where extended defects are present. Extended planar defects of large dimensions like those displayed in Fig.4.a and Fig.5.a can duly modify the propagation of symmetric 6fold vortices. In view of the PFM image in Fig.2.b narrowing or extinction of one of FE domains out of the set in 6-fold vortices would then result.

Strain fields in the lattice change the energy scheme of FE domain walls and cores of vortices. Core fragmentation is assisted by the repulsive potential of the FE domains of the same type ${ }^{31}$. Detailed inspection of Fig.4.b of reference 23 
taken from one $\mathrm{h}-\mathrm{ErMnO}_{3}$ single crystal under mechanical loading during thermal treatment at $10 \mathrm{~K}$ above $\mathrm{T}_{\mathrm{C}}$ and controlled cooling rate, reveals the emergence of FE domains in aligned anti-vortices and patterns of surface poling at the ends of the topological stripes at the edge of the single crystal ${ }^{23}$. Absence of convergence of FE domains in 6-fold vortices to a central point is not rare in h- $\mathrm{RMnO}_{3}$ materials, single crystals of $\mathrm{YMnO}_{3}$ often reveal core fragmentation 8,12,19. The same scenario seems to be repeated in ceramics of the h- $\mathrm{LuMnO}_{3}$ in Fig.4.a. Core fragmentation in 6-fold vortex of DF-TEM image in Fig.4.a occurs where the planar defect crosses the core of the topological vortex. PFM image in Fig.1.b also points to core fragmentation of 6-fold FE vortices of wider scales. Based on the TEM observation core fragmentation can originate from strain fields in the lattice induced by planar defects. Besides core fragmentation, presence of one type of FE domain, $\beta$, right on the planar defect in Fig.4.a and switching of polarization across the defect strikingly display pinning of FE domains to planar defects. In their nature topological defects in h- $\mathrm{RMnO}_{3}$ oxides come from interlocking of FE domain walls and the anti-phase domain walls within less than a unit cell width ${ }^{2}$. Fig.4.a brings in another aspect of the interaction of ferroelectricity and defects with broader domain walls.

Existence of planar defects accompanied by shifts of chemical composition was reported in h- $\mathrm{RMnO}_{3}$ single crystals when oxygen vacancies were present ${ }^{22,31,32,34}$. For the case of the sample in Fig.5 the defective region of the crystalline lattice appears associated with local fluctuations in composition. Analogous observation nearby planar defects were done in sample $\mathrm{x}=0.92$. Selected area electron diffraction (SAED) patterns in defective regions may be sensitive enough to

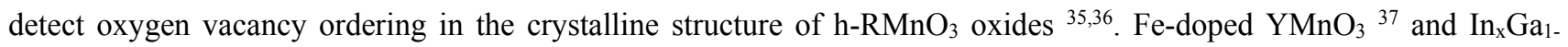
${ }_{x} \mathrm{MnO}_{3}{ }^{38}$ revealed quite complex images of topological defects than those reported for the pure compounds. Ti-doping of $\mathrm{YMnO}_{3}$ also changed formation of topological defects in single crystals ${ }^{39}$. Type-II FE domain networks were reported to be more sensitive than type-I domain network to the chemical gradients in composition like those described in crystalline lattices self-poled by oxygen vacancy gradients ${ }^{31,40}$. Chemical inhomogeneity of the lattice of h- $\mathrm{LuMn}_{\mathrm{x}} \mathrm{O}_{3 \pm \delta}$ bulk ceramics gave rise to new features in topological defects. The hypothetical presence of oxygen vacancies was investigated by looking for the absence of $(1 \overline{1} 1)$ and $(2 \overline{2} 2)$ diffraction spots an indicator of oxygen vacancy ordering. The search in large scale SAED patterns in self-doped h-LuMn $\mathrm{O}_{3 \pm \delta}$ samples of this study gave no evidence of the absence of these diffraction spots neither the FFT of HRTEM images taken from some of the samples provided indication of ordering of oxygen vacancies in the lattice. On the basis of this analysis, one cannot establish that oxygen off-stoichiometry if present in vacancy doped h- $\mathrm{LuMn}_{\mathrm{x}} \mathrm{O}_{3 \pm \delta}$ oxide has concentration high enough to create long range oxygen vacancy ordering of point defects at a detectable threshold in the crystalline structure. Coupling of shifts in chemical composition and planar defects to the formation of FE domains must be considered. In support of this, the PFM analysis in Fig.1.b and $\mathrm{c}$ of $\mathrm{x}=0.92$ sample with highly defective lattice revealed only atypical domain shapes far from those of APB-FE domains in topological type-I or type-II domain networks of the h- $\mathrm{RMnO}_{3}$ single crystals. 
Condensation and annihilation of $\mathrm{FE}$ domain walls in $\mathrm{h}-\mathrm{LuMnO}_{3}$ single crystals under different cooling rates was recently discussed ${ }^{17}$. It was shown that cooling of $\mathrm{LuMnO}_{3}$ through $\mathrm{T}_{\mathrm{C}}(1672 \mathrm{~K})$ resulted in formation of 6-fold vortices, the density of vortices in the $\mathrm{h}-\mathrm{RMnO}_{3}$ being dependent on cooling rate and $\mathrm{R}$ ionic radius ${ }^{5,9,17}$. Heat treating below $\mathrm{T}_{\mathrm{C}}$ e.g. $1671 \mathrm{~K}$ or $1593 \mathrm{~K}$ followed by controlled rate cooling yielded close-loop, annular domains or straight-stripes domain patterns respectively, annihilation of vortex/anti-vortex patterns being observed ${ }^{17}$. Sintering followed by long time annealing at constant temperature of $\mathrm{LuMn}_{\mathrm{x}} \mathrm{O}_{3}$ ceramics in the present study was done at $1573 \mathrm{~K}, 100 \mathrm{~K}$ below the value of $\mathrm{T}_{\mathrm{C}}$ reported above. At the comparatively low process temperature of $94 \%$ of $\mathrm{T}_{\mathrm{C}}$ of $\mathrm{LuMnO}_{3}$ low density straightstripe patterns would be expected in the crystalline grains of the bulk ceramic. The current investigation on self-doping of $\mathrm{h}-\mathrm{RMn}_{\mathrm{x}} \mathrm{O}_{3}$ ceramics indicates that dealing with ceramics where grain boundaries and planar defects play key roles on properties the condensation and annihilation mechanism of FE vortex/antivortex patterns needs to be reexamined. Keeping constant the cooling rate for $\mathrm{LuMn}_{\mathrm{x}} \mathrm{O}_{3}$ bulk materials processed below $\mathrm{T}_{\mathrm{C}}$ one observed vortex/antivortex patterns in these ceramics where only the nominal vacancy contents and annealing time were variables. The microstructural study of same samples has proven that these two parameters are major factors governing the final grain size and overall quality of $\mathrm{LuMn}_{\mathrm{x}} \mathrm{O}_{3}$ ceramics. The main driving force for creation of vortices below $\mathrm{T}_{\mathrm{C}}$ has to be looked for on fluctuations of chemical potential during growth of grains. This hypothesis can be linked to the observation of FE vortices/circles in synthesized $\mathrm{YMnO}_{3}$ and $\mathrm{YMn}_{0.9} \mathrm{Fe}_{0.1} \mathrm{O}_{3}$ particles below $\mathrm{T}_{\mathrm{C}}{ }^{37}$. The matter of condensation/annihilation of vortex/antivortex upon cooling above or below $\mathrm{T}_{\mathrm{C}}$ in bulk $\mathrm{RMnO}_{3}$ ceramics deserves further study. The role of chemistry shifts in the lattice on creation of different patterns of FE domains like vortices, stripes or circular domains below $\mathrm{T}_{\mathrm{C}}$ should be further examined in single crystals of $\mathrm{h}-\mathrm{RMnO}_{3}$ oxides whenever off-stoichiometric on either Mn site or rare-earth site is introduced.

FE domains and topological defects in $\mathrm{h}-\mathrm{LuMn}_{\mathrm{x}} \mathrm{O}_{3 \pm \delta}$ bulk ceramics with the lattice altered by the $\mathrm{Mn} / \mathrm{Lu}$ ratio were investigated here. Patterns of complete 6 -fold vortices in different sizes were found upon change of annealing time or introducing $\mathrm{Lu}$ vacancies. It is found that $\mathrm{Lu}$ vacancy doping led to large FE domains as fast grain growth on the $\mathrm{x}>1.00$ side of the h-LuMn $\mathrm{O}_{3 \pm \delta}$ solid solution yields dense microstructures with coarse grain sizes. Large 6-fold vortices of size as large as $20 \mu \mathrm{m}$ comparable to those found in single crystals could be replicated by h-LuMn $\mathrm{O}_{3 \pm \delta}$ ceramics via $\mathrm{Lu}$ vacancy doping and enlarging the grain size by increasing annealing time at $1300{ }^{\circ} \mathrm{C}$. Grain boundaries and planar defects inside crystalline grains were identified as common 6-fold vortex topology breaking features. Nano-vortices were revealed by TEM microscopy where pinning of FE domains to planar defects was observed. FE polarization switching determined in HRTEM images reveals that planar defects or chemical inhomogeneity inside crystalline grains play a major role in ferroelectricity. SAED patterns of electron diffraction fail to substantiate oxygen vacancy ordering in the crystalline lattice of self-doped samples, although the FE domain network was observed in PFM images of Mn vacancy 
doped samples. Poling followed by reversed poling showed that the memory effect exists for the FE domains in offstoichiometric ceramics of h- $\mathrm{LuMn}_{\mathrm{x}} \mathrm{O}_{3 \pm \delta}$ solid solution.

\section{Supplementary Materials}

See supplementary material for change in the volume of the unit cell of sintered ceramics versus $\mathrm{x}$ at different annealing time (Figure A1), complementary AFM and PFM images of some figures discussed in the text (Figure A2, Figure A3, Figure A4), as were addressed in the text.

\section{ACKNOWLEDGMENTS}

A.B would acknowledge the financial support of FCT fellowships SFRH/BD/51140/2010. We would like to acknowledge the Electron Microscopy Group in Fritz Habor Institute, Berlin for providing the Equipment for TEM Studies. A.B acknowledges Achim Klein-Hoffmann for teaching sample preparation and his supports. Also, it would be acknowledged the help of staff in Microscopy Center of the Aveiro University, Portugal and the FCT project REDE/1509/RME/2005 providing access to electron microscopy facilities in CICECO, and also PFM equipment in CICECO.

\section{References}

${ }^{1}$ D. Meier, M. Lilienblum, P. Becker, L. Bohaty, N.A. Spaldin, R. Ramesh, M. Fiebig, and L. Bohatý, Phase Transitions 86, 33 (2013).

${ }^{2}$ S. Chae, Y. Horibe, D. Jeong, N. Lee, K. Iida, M. Tanimura, and S.-W. Cheong, Phys. Rev. Lett. 110, 167601 (2013).

${ }^{3}$ Y. Kumagai and N.A. Spaldin, Nat. Commun. 4, 1 (2013).

${ }^{4}$ H. Das, A.L. Wysocki, Y. Geng, W. Wu, and C.J. Fennie, Nat. Commun. 5, 1 (2014).

${ }^{5}$ S.M. Griffin, M. Lilienblum, K.T. Delaney, Y. Kumagai, M. Fiebig, and N.A. Spaldin, Phys. Rev. X 2, 41022 (2012).

${ }^{6}$ J. Li, F.-K. Chiang, Z. Chen, C. Ma, M.-W. Chu, C.-H. Chen, H. Tian, H. Yang, and J. Li, Sci. Rep. 6, 28047 (2016).

${ }^{7}$ Q.-H. Zhang, G.-T. Tan, L. Gu, Y. Yao, C.-Q. Jin, Y.-G. Wang, X.-F. Duan, and R.-C. Yu, Appl. Phys. Lett. 105, 12902 (2014).

${ }^{8}$ L. Tian, Y. Wang, B. Ge, X. Zhang, and Z. Zhang, Appl. Phys. Lett. 106, 112903 (2015).

${ }^{9}$ M. Lilienblum, T. Lottermoser, S. Manz, S.M. Selbach, A. Cano, and M. Fiebig, Nat. Phys. 11, 1070 (2015).

${ }^{10}$ S. Artyukhin, K.T. Delaney, N.A. Spaldin, and M. Mostovoy, Nat. Mater. 13, 42 (2014).

${ }^{11}$ S.C. Chae, N. Lee, Y. Horibe, M. Tanimura, S. Mori, B. Gao, S. Carr, and S.-W.W. Cheong, Phys. Rev. Lett. 108, 167603 (2012).

${ }^{12}$ T. Jungk, Á. Hoffmann, M. Fiebig, E. Soergel, A. Hoffmann, M. Fiebig, and E. Soergel, Appl. Phys. Lett. 97, 12904 (2010).

${ }^{13}$ B. Mettout, P. Tolédano, M. Lilienblum, and M. Fiebig, Phys. Rev. B 89, 24103 (2014).

${ }^{14}$ T. Jungk, Á. Hoffmann, and E. Soergel, New J. Phys. 10, 13019 (2008). 
${ }^{15}$ B.J. Rodriguez, S. Jesse, A.P. Baddorf, and S. V. Kalinin, Phys. Rev. Lett. 96, 237602 (2006).

${ }^{16}$ T. Choi, Y. Horibe, H.T. Yi, Y.J. Choi, W. Wu, and S.-W. Cheong, Nat. Mater. 9, 253 (2010).

${ }^{17}$ S.-Z. Lin, X. Wang, Y. Kamiya, G.-W. Chern, F. Fan, D. Fan, B. Casas, Y. Liu, V. Kiryukhin, W.H. Zurek, C.D. Batista, and S.-W. Cheong, Nat. Phys. 10, 970 (2014).

${ }^{18}$ Q. Zhang, G. Tan, L. Gu, Y. Yao, C. Jin, Y. Wang, X. Duan, and R. Yu, Sci. Rep. 3, 2741 (2013).

${ }^{19}$ Y. Yu, X. Zhang, Y.G. Zhao, N. Jiang, R. Yu, J.W. Wang, C. Fan, X.F. Sun, and J. Zhu, Appl. Phys. Lett. 103, 32901 (2013).

${ }^{20}$ A. del Campo, A. Retzker, and M.B. Plenio, New J. Phys. 13, 83022 (2011).

${ }^{21}$ M.S. Vijaya Kumar, N. Higaki, K. Kuribayashi, T. Hibiya, and S. Yoda, J. Am. Ceram. Soc. 94, 281 (2011).

${ }^{22}$ S.C. Chae, Y. Horibe, D.Y. Jeong, S. Rodan, N. Lee, and S.-W. Cheong, Proc. Natl. Acad. Sci. U. S. A. 107, 21366 (2010).

${ }^{23}$ X. Wang, M. Mostovoy, M.G. Han, Y. Horibe, T. Aoki, Y. Zhu, and S.-W. Cheong, Phys. Rev. Lett. 112, 1 (2014).

${ }^{24}$ D. Hull and D.J. Bacon, Introduction to Dislocations (Butterworth-Heinemann, 2011).

${ }^{25}$ T. Lonkai, D.G. Tomuta, U. Amann, J. Ihringer, R.W.A. Hendrikx, D. Többens, J.A. Mydosh, D.M. Tobbens, and J.A. Mydosh, Phys. Rev. B - Condens. Matter Mater. Phys. 69, 134108 (2004).

${ }^{26}$ H. Sim, J. Oh, J. Jeong, M.D. Le, and J.G. Park, Acta Crystallogr. B. Struct. Sci. Cryst. Eng. Mater. 72, 3 (2016).

27 T. Katsufuji, S. Mori, M. Masaki, Y. Moritomo, N. Yamamoto, and H. Takagi, Phys. Rev. B 64, 104419 (2001).

${ }^{28}$ M. Lilienblum, E. Soergel, and M. Fiebig, J. Appl. Phys. 110, 52007 (2011).

${ }^{29}$ B.A. Almeida, M.C. Ferro, A. Ravanan, P.M.F. Grave, H.-Y. Wu, M.-X. Gao, Y. Pan, F.J. Oliveira, A.B. Lopes, and J.M. Vieira, IOP Conf. Ser. Mater. Sci. Eng. 55, 12001 (2014).

${ }^{30}$ A. Baghizadeh, J.M. Vieira, J.S. Amaral, M.P. Graça, M.R. Soares, D.A. Mota, and V.S. Amaral, J. Magn. Magn. Mater. 395, 303 (2015).

${ }^{31}$ X. Wang, F.-T. Huang, R. Hu, F. Fan, and S. Cheong, APL Mater. 3, 41505 (2015).

${ }^{32}$ Y. Du, X. Wang, D. Chen, Y. Yu, W. Hao, Z. Cheng, and S.X. Dou, Phys. Chem. Chem. Phys. 15, 20010 (2013).

${ }^{33}$ Y. Du, X.L. Wang, D.P. Chen, S.X. Dou, Z.X. Cheng, M. Higgins, G. Wallace, and J.Y. Wang, Appl. Phys. Lett. 99, 252107 (2011).

${ }^{34}$ D.P. Chen, Y. Du, X.L. Wang, Z.X. Cheng, S.X. Dou, Z.W. Lin, J.G. Zhu, and B. Xu, J. Appl. Phys. 111, 53 (2012).

${ }^{35}$ S. Cheng, S.Q. Deng, Y.G. Zhao, X.F. Sun, and J. Zhu, Appl. Phys. Lett. 106, 62905 (2015).

${ }^{36}$ Q.H. Zhang, X. Shen, Y. Yao, Y.G. Wang, C.Q. Jin, and R.C. Yu, J. Alloys Compd. 648, 253 (2015).

${ }^{37}$ M.H. Harunsani, J.Q. Li, Y.B. Qin, H.T. Tian, J.Q. Li, H.X. Yang, and R.I. Walton, Appl. Phys. Lett. 107, 62905 (2015).

${ }^{38}$ F.-T. Huang, X. Wang, S.M. Griffin, Y. Kumagai, O. Gindele, M.-W. Chu, Y. Horibe, N.A. Spaldin, and S.-W. Cheong, Phys. Rev. Lett. 113, 267602 (2014).

${ }^{39}$ S. Mori, K. Kurushima, H. Kamo, Y. Ishii, F.-T. Huang, Y. Horibe, T. Kastufuji, and S.-W. Cheong, Ferroelectrics 462, 50 (2014).

${ }^{40}$ F. Xue, X. Wang, I. Socolenco, Y. Gu, L.-Q. Chen, and S.-W. Cheong, Sci. Rep. 5, 17057 (2015). 


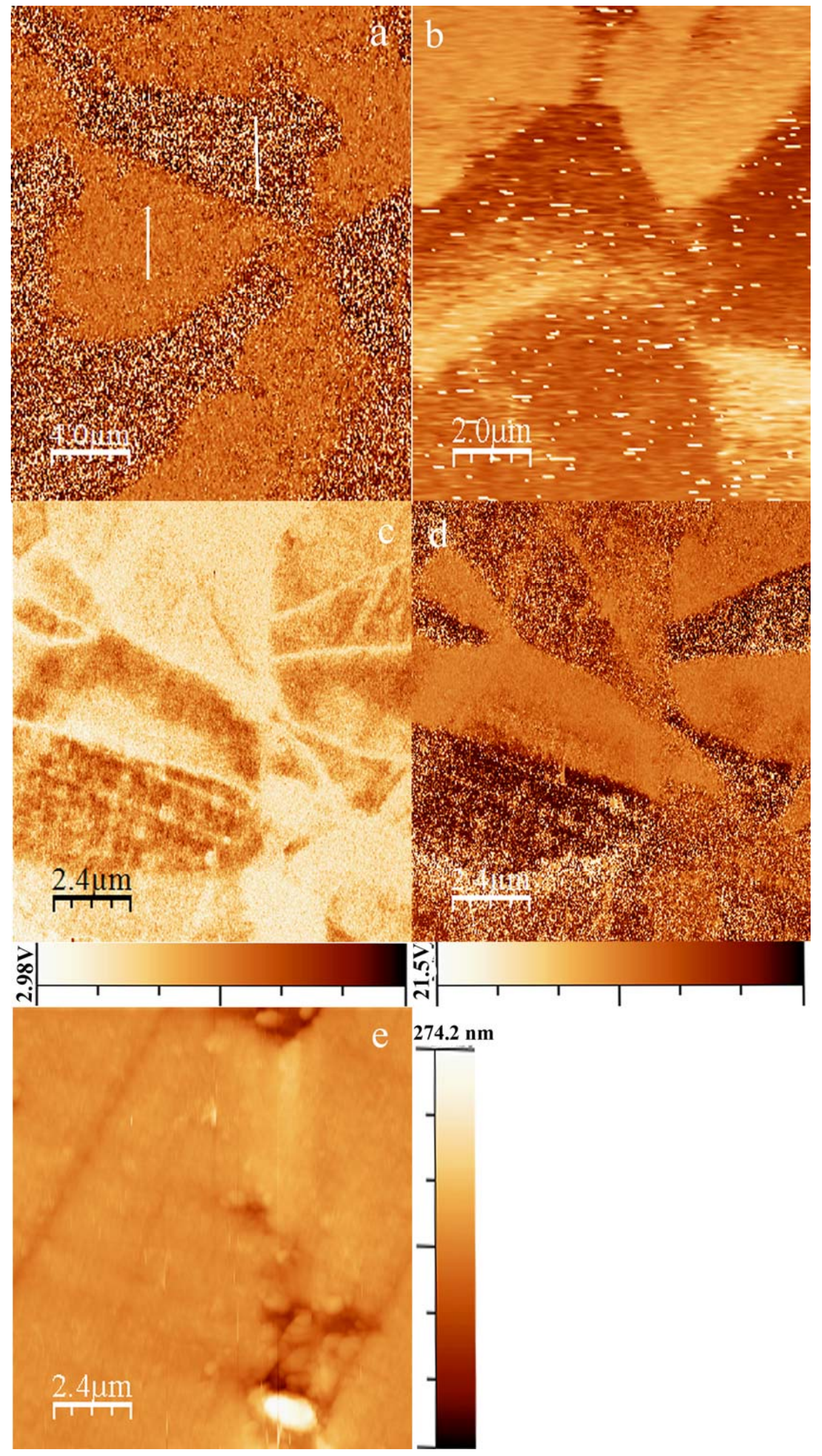


FIG. 1. (a) PFM image of 6-fold vortices in sample $x=1.04$ (10 days annealing) and 20 microns wide. (b) PFM image of 6-fold vortices of $x=1.00$ stoichiometric sample after 5 days annealing with size of less than 10 microns. The topography map of figures (a) and (b) are provided in Supplementary Materials, Figure A2. FE domains of sample $x=0.92$ (10 days annealing), (c) amplitude map and (d) phase map, where the left side of the images show 6-fold vortices and the right side, broken 6-fold symmetry upon meeting the grain boundary. (e ) The topography map of (c) and (d). 


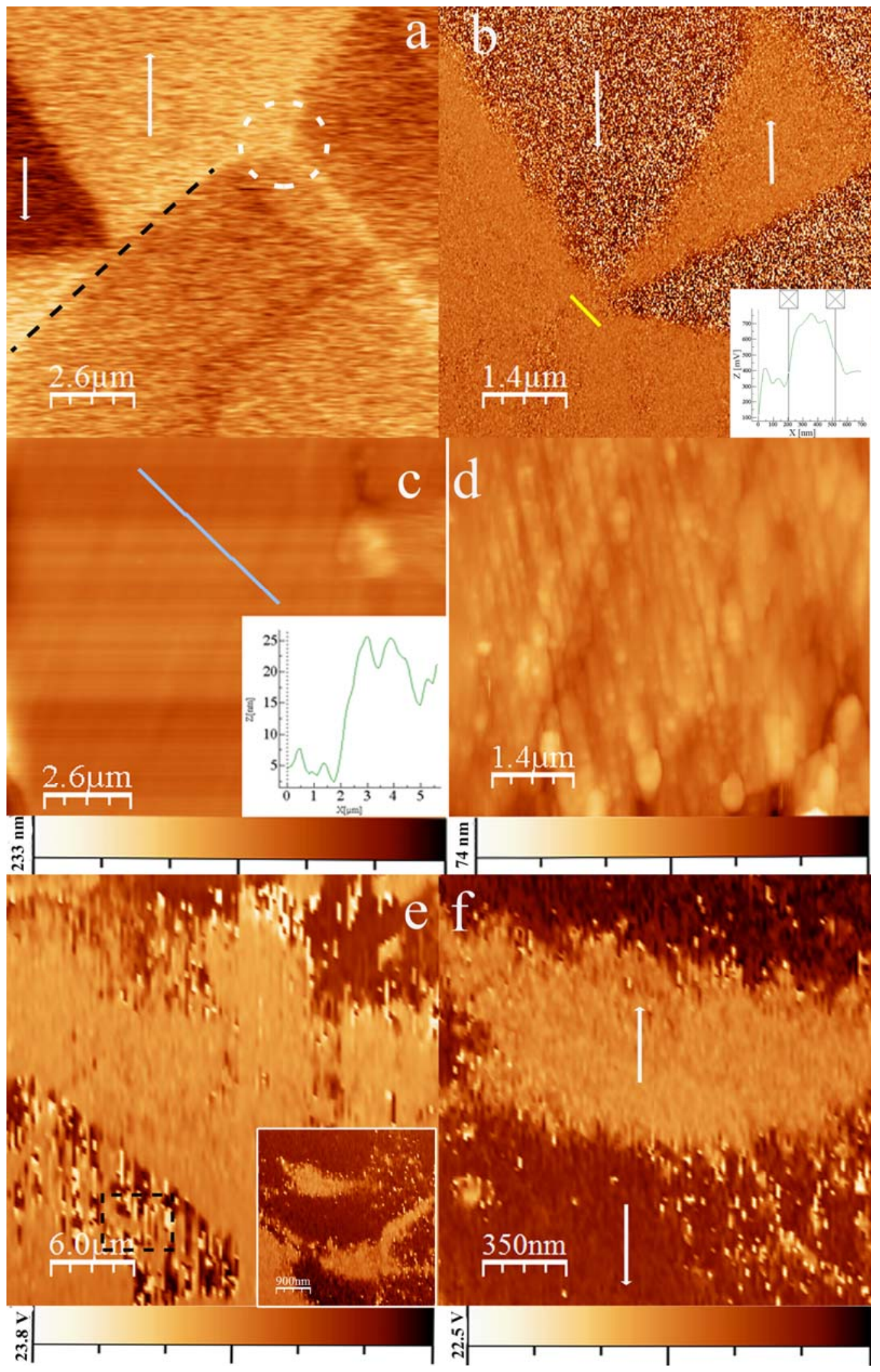

FIG. 2. (a) and (c) Phase and topography maps of a broken 6-fold symmetry of sample $x=1.04$ (5 days annealing), the dark-dashed line shows the grain border where topology breaking occurs and the white circle gives the triple line of three grain-boundaries. The light, blue line in (c) shows the location of grain boundary where in the inset of the image, change in the height profile indicates sudden step. (b) and (d) Phase and topography maps of topology breaking in sample $x=1.00$ ( 5 days annealing) where missing of one of the FE domain with downward polarization is noted; variation in voltage across yellow line is given as inset, the distance between two markers is $290 \mathrm{~nm}$. (e) Large FE domain with upward polarization seen in sample $\mathrm{x}=1.04$ (10 days annealing) revealing dispersed FE 
nano-domains of opposite polarization, with black-dashed box illustrating the part of image shown as inset, (f) enlarged image of inset in (e) confirms the existence of nano-FE domains in image (e). (Figure A4, Supplementary Materials show additional analysis of the same region)
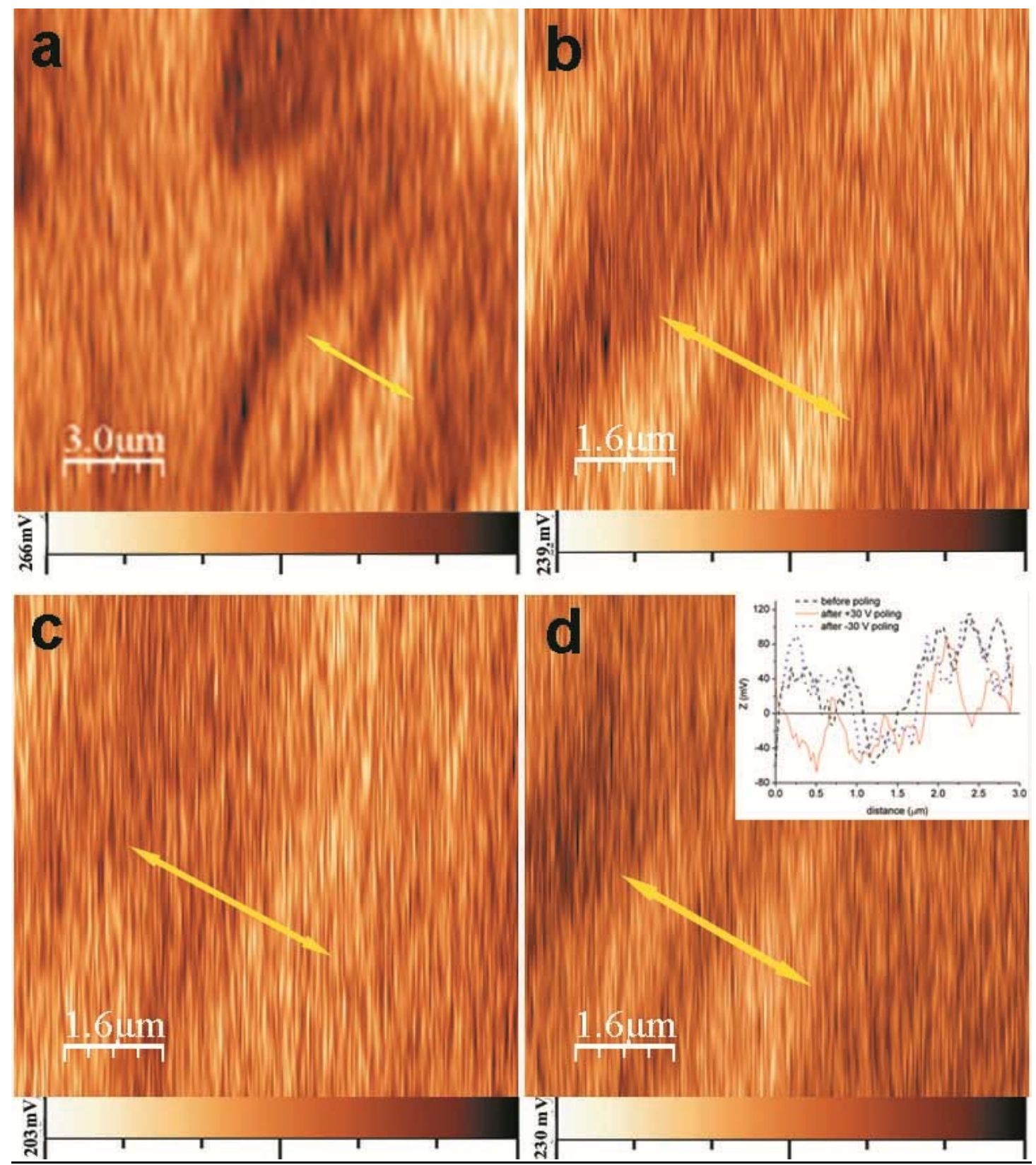

FIG 3. (a). PFM image of sample $x=1.04$ (10 days annealing) showing FE domains of negative polarization (dark contrast) and positive (bright contrast). (b) Area selected for electrical poling experiment, (c) after applying $+30 \mathrm{~V}$ poling voltage and d) after applying $-30 \mathrm{~V}$ voltage shows the same FE domain initially seen in Fig.3.b. The inset gives the intensity profile of the voltage across the arrowed lines before and after poling. 


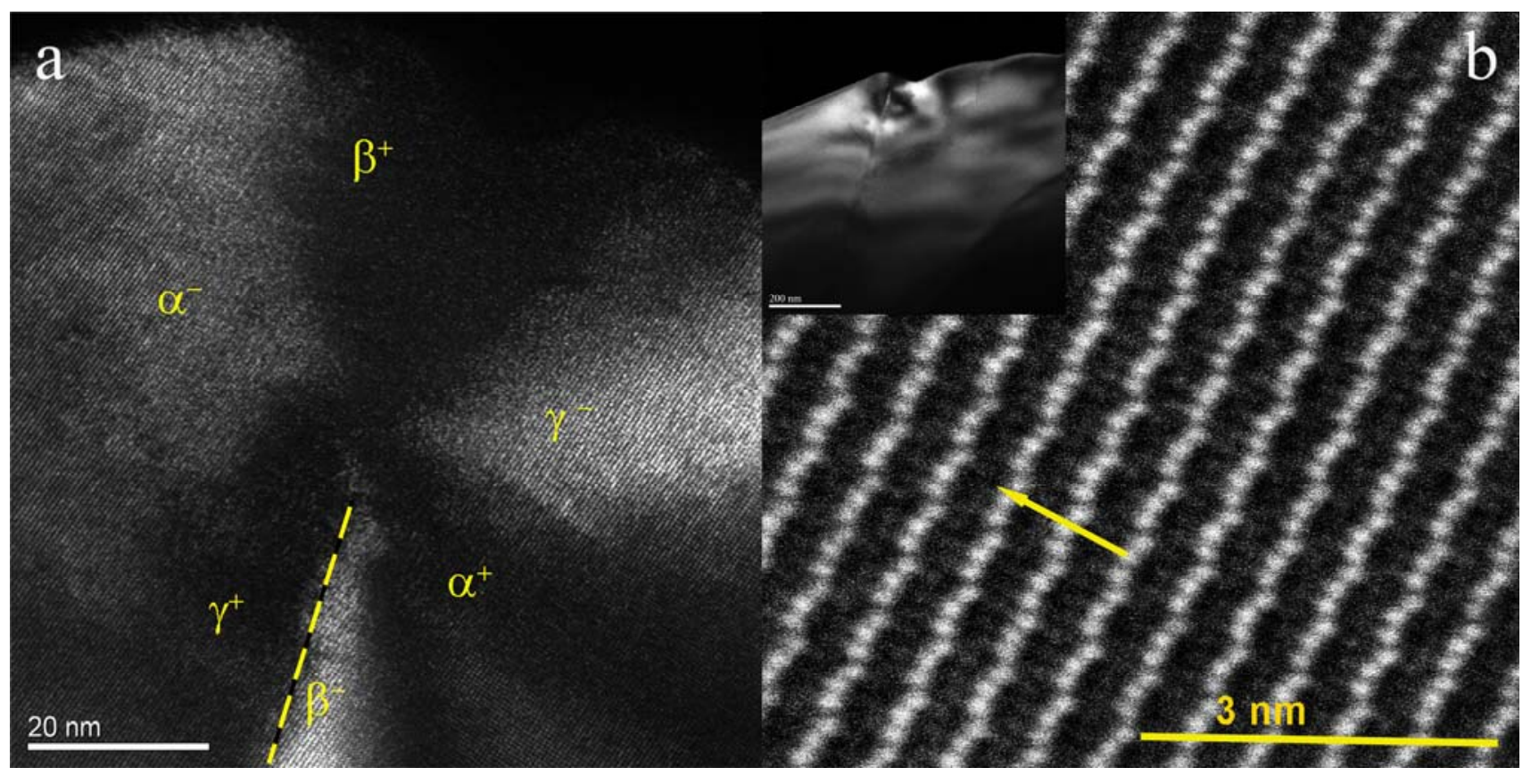

FIG. 4. (a) DF-TEM image of crystalline grain of sample $x=1.04$ (10 days annealing) aligned to $<110>$ zone axis with a 6 -fold vortex of nano-dimensions on the edge of the particle, with the antiphase shift of FE domains. The dash-line is showing the approximate location of the planar defect propagated into the vortex-core. (004) plane of diffraction pattern was used to take dark field images.

(b) The HAADF-STEM image of the same lattice area of (a) showing the electrical polarization direction (the arrow) corresponding to displacement of the Lu ions; inset, low magnification TEM dark field image of same area of the crystalline grain with an extended planar defect propagated from the edge to the inside of the particle. 


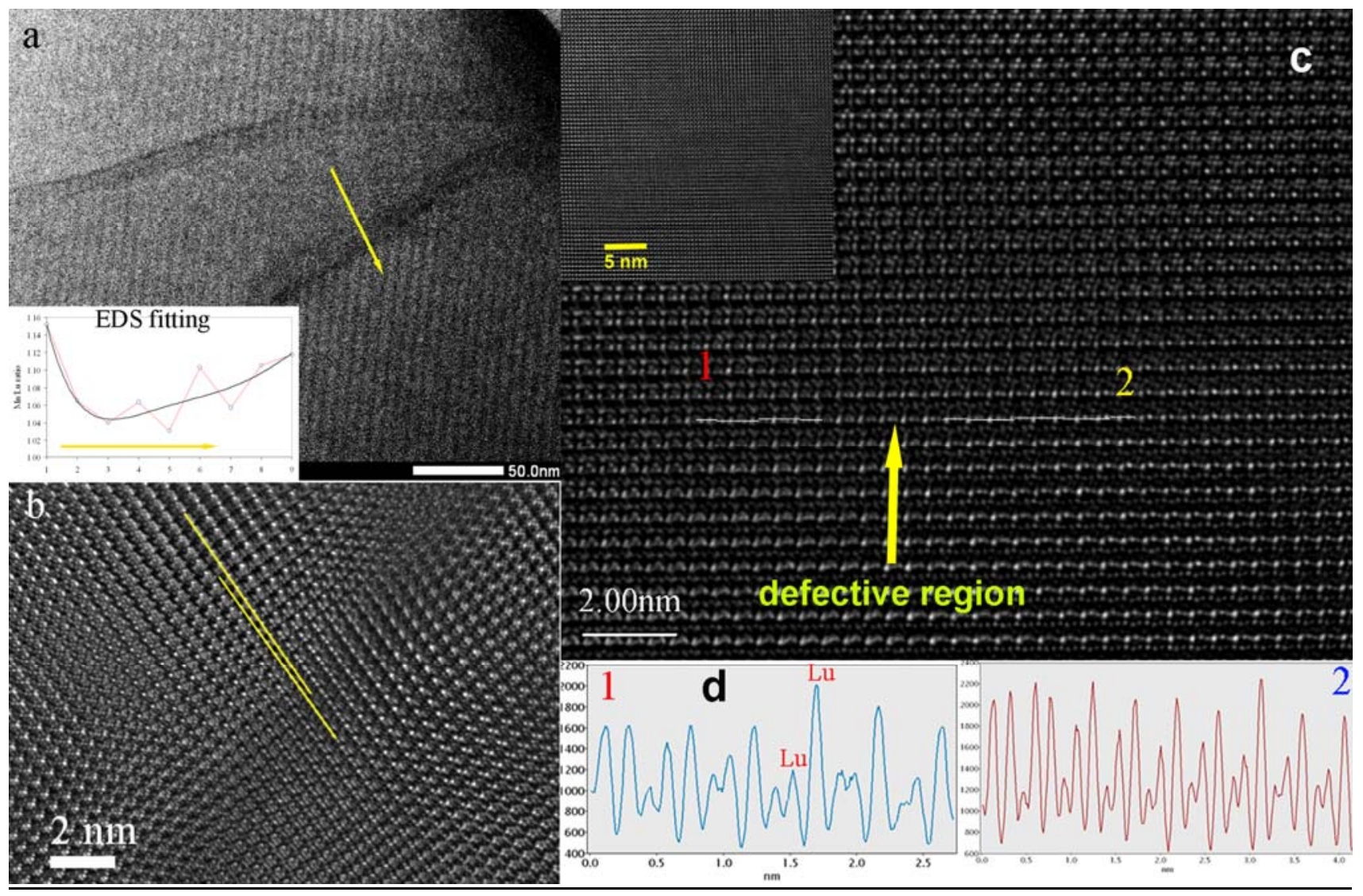

FIG. 5. (a) Sample $x=1.04$, (10 days annealing), aligned to $<110>$ zone axis, two planar defects emerging from one same core with EDS analysis across of the defects as inset. (b) HRTEM image of the same region taken from one of the planar defects displaying antiphase boundary like structure. (c) HRTEM image of sample $x=1.00$ ( 5 days annealing) showing arrangements of Lu ions around a defect shown as inset of (c), the white lines marked 1 and 2 were used to determine the profile intensities used to identify the position of atomic planes with FE switching and antiphase shift on the domain wall in (d). 